\title{
Correlation between chromosomal polymorphisms and male infertility in a Northeast Chinese population
}

L.L. Li' ${ }^{1}$, D. Peng ${ }^{1}$, R.X. Wang ${ }^{1}$, H.B. Zhu' ${ }^{1}$, W.J. Wang ${ }^{2}$ and R.Z. Liu ${ }^{1}$

${ }^{1}$ Center for Reproductive Medicine of the First Bethune Hospital of Jilin University, Changchun, Jilin, China

'Department of Medical Genetics,College of Basic Medical Sciences, Jilin University, Changchun, Jilin, China

Corresponding author: R.Z. Liu

E-mail: Irz410@126.com

Genet. Mol. Res. 14 (4): 15435-15443 (2015)

Received March 26, 2015

Accepted July 2, 2015

Published November 30, 2015

DOI http://dx.doi.org/10.4238/2015.November.30.21

ABSTRACT. The aim of this study was to evaluate the correlation between chromosomal polymorphisms and male infertility. The patients were diagnosed with azoospermia or oligospermiaby a semen analysis. Chromosomal analysis was performed on peripheral blood lymphocytes obtained from the patients, with standard G-banding and C-banding. Y chromosome microdeletions were detected by multiplex polymerase chain reaction (PCR) amplification. The parents of 35 polymorphic probands were also subjected to chromosomal analysis, and their detailed reproductive histories were surveyed. The frequency of autosomal polymorphisms did not differ significantly among the infertile patients and fertile control individuals. The frequency of the Yqh-variant increased with the decrease in sperm count; this appeared at a significantly higher frequency in the azoospermia group (57.2 vs 24.3 vs $0 \%$ ). The results of PCR amplification indicated that $32.14 \%$ of the patients with Yqh \pm had microdeletions in the $Y$ chromosome. The parents of the probands with the same chromosomal polymorphisms as the probands (among the 35 recalled families) did not show any adverse 
reproductive history. We observed no significant correlations between autosomal polymorphisms and male infertility. However, we observed a significant increase in the frequency of Yqh- in the azoospermic patients. This may be attributed to $Y$ chromosome microdeletions, although the association between $Y$ chromosome microdeletions and $Y$ chromosome variants remains to be elucidated.

Key words: Chromosomal polymorphisms; Male infertility; Azoospermia; Pedigree analysis; Y chromosome microdeletion

\section{INTRODUCTION}

Male-factor infertility is a marital problem that is impossible to ignore. It accounts for approximately $50 \%$ of the couple infertility cases (Poongothai et al., 2009). Most cases of nonobstructive azoospermia or severe oligospermia result from idiopathic causes of spermatogenetic failure (Irvine, 2002). Therefore, the study of human chromosomes plays a key role in the diagnosis, prognosis, treatment, and monitoring of infertility problems.

Early published studies have confirmed that the incidence of chromosomal abnormalities in people with infertility was greater than the overall incidence of chromosomal abnormalities in the general population (Mierla and Stoian, 2012). However, the association between chromosomal polymorphic variants and male infertility remains to be undefined. Polymorphic variants, particularly in the polymorphic region of chromosomes $1,9,16$, and $\mathrm{Y}$, and the nucleolar organizing region (NOR) of acrocentric chromosomes, occur in the general population (Schaffer and Tommerup, 2005). Variants, such as 9qh+, Yqh+ and D/G group are very common findings in routine cytogenetics, with frequencies of approximately $2.44 \%$, approximately $2.85 \%$ and approximately $3.96 \%$, respectively (Bhasin, 2005). Although chromosomal polymorphisms have been categorized as minor chromosomal rearrangements that do not correlate with abnormal phenotypes, many previous reports have presented conflicting views regarding its association with infertility (Madon et al., 2005).

In this study, 2584 infertile patients were subjected to cytogenetic and semen analysis, in order to evaluate the correlation between polymorphisms in the chromosomal heterochromatin and male infertility. The possible influence of chromosomal polymorphisms on infertility was evaluated in 35 polymorphic patients and their parents (who displayed no adverse reproductive history).

\section{MATERIAL AND METHODS}

\section{Patients}

Male patients who sought infertility management at the Center for Reproductive Medicine of the First Bethune Hospital of Jilin University between January 2008 and October 2013 ( $N=$ 2584) were included in the study group, while 602 random individuals were recruited into the control group I. Control groups II, III, and IV, on the other hand, comprised fertile male individuals with a normal karyotype, azoospermic patients with a normal karyotype, and oligospermic patients with a normal karyotype ( $\mathrm{N}=50$ each).

\section{Chromosomal analysis}

The patient chromosomes (obtained from peripheral blood lymphocytes) were subjected 
to cytogenetic investigations. The lymphocytes were cultured in RPMI 1640 (Gibco; Invitrogen, Carlsbad, CA, USA), supplemented with $0.5 \mathrm{mg} / \mathrm{mL}$ phytohemagglutinin (Shanghai Yihua Medical Technology Co., Ltd., Shanghai, China), and fetal bovine serum to the final concentration of $20 \%$ (Beijing Dingguo Biotechnology, Beijing, China) for $72 \mathrm{~h}$, followed by treatment with $50 \mu \mathrm{g} / \mathrm{mL}$ colcemid (BaiDi Bio-Technology Co., Ltd.,China). Metaphase chromosome spreads were studied by standard GTG ( $\mathrm{G}$ bands by trypsin using Giemsa) and CBG ( $\mathrm{C}$ bands by bariumhydroxide using Giemsa) banding procedures (Dong et al., 2012), which included the use of trypsin and Giemsa for G-banding and barium hydroxide for C-banding.

\section{Semen analysis}

Semen samples were obtained from the study subjects for analysis after 2-7 days of sexual abstinence. The liquefied samples were analyzed thrice, according to standard guidelines (World Health Organization, 2010). Sperm count below $15 \times 10^{6} / \mathrm{mL}$ was defined as oligospermia, while the absence of sperms in the ejaculate, or the sample after centrifugation, was defined as azoospermia.

\section{Multiplex polymerase chain reaction (PCR) amplification}

Genomic DNA was isolated from peripheral blood lymphocytes using a blood DNA extraction mini kit (Tiangen Biotech Co., Ltd., Beijing, China).

Microdeletions in the $Y$ chromosome were screened by multiplex PCR. A series of 9 specific STSs (sequence-tagged sites), mapped in the AZF region, and human zinc-finger proteinencoding genes (ZFXIZFY) located on the $X$ and $Y$ chromosomes were selected for analysis. The $Z F X I Z F Y$, acting as internal control primers, were selected for molecular genetic analysis of deletion. The specific STSs included SY84 and SY86 for AZFa, SY27, SY134, and SY143 for AZFb, and SY152, SY157, SY254, and SY255 for AZFc.

Multiplex PCR amplification was performed in a 10- $\mu \mathrm{L}$ reaction system, containing 200 ng genomic DNA,1.5 mM Mg${ }^{2+}, 800 \mu \mathrm{M}$ dNTP, $10 \mathrm{pM}$ of each primer, and $2 \mathrm{U}$ Taq polymerase. Thermocycling (Applied Biosystems, Inc., Foster City, CA, USA) consisted of an initial denaturation at $94^{\circ} \mathrm{C}$ for $6 \mathrm{~min}, 35 \mathrm{cycles}$ at $94^{\circ} \mathrm{C}$ for $40 \mathrm{~s}, 55^{\circ} \mathrm{C}$ for $45 \mathrm{~s}$, and $72^{\circ} \mathrm{C}$ for $60 \mathrm{~s}$, and a final extension step at $72^{\circ} \mathrm{C}$ for $6 \mathrm{~min}$. Men without microdeletions in the $\mathrm{Y}$ chromosome and women were used as positive and negative controls to ensure the performance of the amplification reaction. In addition, blank controls were employed to ensure that the samples were not contaminated during processing. The products were finally stored at $4^{\circ} \mathrm{C}$ prior to electrophoretic detection.

PCR products $(8 \mu \mathrm{L})$ mixed with $6 \mathrm{X}$ loading buffers $(1-2 \mu \mathrm{L})$ were separated on a $1.5 \%$ agarose gel (LP0028A; Oxoid, UK) containing ethidium bromide $(0.5 \mu \mathrm{g} / \mathrm{mL})$, at $120 \mathrm{~V}$ for $25 \mathrm{~min}$. The samples showing STS deletions were further confirmed by a repeated round of testing (as described above).

\section{Statistical analysis}

The SPSS v.17.0 software platform (IBM, Armonk, NY, USA) was used to analyze all data. All descriptive statistics are reported as means \pm standard deviation or ratio. Independent sample $t$-tests were used to test all numerical data. The Pearson chi-square test was used to test the categorical data. All $P$ values were two-sided, and $P<0.05$ was considered to be statistically significant. 


\section{RESULTS}

Chromosomal polymorphisms were observed in 101/2584 infertile patients and 19/602 random individuals; the frequency was not statistically significant (3.91 vs 3.16\%). The chromosomal polymorphic variants were divided into four sections: 1/9/16qh \pm , variants in the NOR of acrocentric chromosomes (D/G group), pericentric inversion of chromosome 9, and Yqh \pm . The frequencies and constituent ratio of the four types of chromosomal polymorphic variants in the study group and control group I are summarized in Table 1.

Table 1. Prevalence and proportion of chromosomal polymorphic variants in the study group and control group I.

\begin{tabular}{|c|c|c|c|c|c|c|}
\hline \multirow[t]{2}{*}{ Variants } & \multicolumn{3}{|c|}{ Study Group } & \multicolumn{3}{|c|}{ Control Group I } \\
\hline & Cases & Prevalence & Proportion & Cases & Prevalence & Proportion \\
\hline $1 / 9 / 16 q h \pm$ & 15 & $0.58 \%(15 / 2584)$ & $14.9 \%(15 / 101)$ & 4 & $0.66 \%(4 / 602)$ & $21.1 \%(4 / 19)$ \\
\hline D/G group & 17 & $0.66 \%(17 / 2584)$ & $16.8 \%(17 / 101)$ & 4 & $0.66 \%(4 / 602)$ & $21.1 \%(4 / 19)$ \\
\hline Inv (9) & 17 & $0.66 \%(17 / 2584)$ & $16.8 \%(17 / 101)$ & 6 & $1.00 \%(6 / 602)$ & $31.5 \%(6 / 19)$ \\
\hline Yqh \pm & 52 & $2.01 \%(52 / 2584)^{*}$ & $51.5 \%(52 / 101)^{*}$ & 5 & $0.84 \%(5 / 602)$ & $26.3 \%(5 / 19)$ \\
\hline Total & 101 & $3.91 \%(101 / 2584)$ & $100 \%(101 / 101)$ & 19 & $3.16 \%(19 / 602)$ & $100 \%(19 / 19)$ \\
\hline
\end{tabular}

${ }^{*}$ Compared with control group I, $\mathrm{P}<0.05$.

The Pearson chi-square analysis revealed no significant differences in the frequency of autosome variants between the study group and control group I. However, the frequency of chromosome $\mathrm{Y}$ variants in the study group was significantly increased compared to the control group (2.01 vs $0.84 \%)$. The frequency of chromosome $Y$ variants was much higher in the study group than the three types of autosomal variants, while the frequencies of the four types of chromosomal polymorphic variants were similar in the control group.

Ninety-two of 101 infertile patients with chromosomal polymorphisms were subjected to semen analysis. The results summarized in Table 2 indicated that 42, 37, and 13 patients displayed azoospermia, oligospermia, and a normal sperm count, respectively.

Table 2. Comparison of the cases of chromosomal polymorphic variants in the groups of different sperm count.

\begin{tabular}{|c|c|c|c|c|c|}
\hline Variants & Karyotype & Azoospermia & Oligospermia & Normal sperm count & Cases \\
\hline \multirow[t]{3}{*}{ 1/9/16qh \pm} & $46, \mathrm{XY}, 1 \mathrm{qh}+$ & 2 & 2 & 3 & 7 \\
\hline & $46, \mathrm{XY}, 9 \mathrm{qh}+$ & 1 & 0 & 0 & 1 \\
\hline & 46,XY,16qh+ & 0 & 3 & 1 & 4 \\
\hline \multirow[t]{12}{*}{ D/G Group } & 46,XY,13pstk+ & 1 & 0 & 0 & 1 \\
\hline & 46,XY,13pstk- & 0 & 1 & 0 & 1 \\
\hline & $46, X Y, 13 p+$ & 0 & 1 & 0 & 1 \\
\hline & $46, \mathrm{XY}, 14 \mathrm{pstk}+$ & 1 & 0 & 1 & 2 \\
\hline & $46, \mathrm{XY}, 14$ pstk- & 0 & 0 & 1 & 1 \\
\hline & $46, X Y, 14 p+$ & 0 & 1 & 0 & 1 \\
\hline & 46,XY,15pstk- & 1 & 0 & 0 & 1 \\
\hline & $46, X Y, 15 p-$ & 0 & 1 & 0 & 1 \\
\hline & 46,XY,21pstk+ & 0 & 2 & 0 & 2 \\
\hline & $46, X Y, 21$ pstk- & 0 & 2 & 0 & 2 \\
\hline & $46, X Y, 21 p s s$ & 1 & 0 & 0 & 1 \\
\hline & $46, X Y, 22$ pstk+ & 0 & 1 & 0 & 1 \\
\hline \multirow[t]{2}{*}{ inv (9) } & $46, X Y, \operatorname{inv}(9),(p 11 q 12)$ & 3 & 6 & 1 & 10 \\
\hline & $46, X Y, \operatorname{inv}(9),(p 11 q 13)$ & 2 & 0 & 3 & 5 \\
\hline \multirow[t]{2}{*}{ Yqh \pm} & $46, \mathrm{XY}, \mathrm{qh}+$ & 6 & 8 & 3 & 17 \\
\hline & $46, \mathrm{XY}, \mathrm{qh}-$ & 24 & 9 & 0 & 33 \\
\hline Total & & 42 & 37 & 13 & 92 \\
\hline
\end{tabular}


Moreover, the Yqh \pm variant was observed in a significantly high frequency in the patients displaying azoospermia (especially the Yqh- variant). A majority of the patients displaying azoospermia (30/42; 71.4\%) presented the Yqh \pm variant, while this variant was present in only $23.1 \%(3 / 13)$ of the patients displaying a normal sperm count. The frequency of the Yqh- variant was increased with the decrease in sperm count; a significantly high frequency of this variant was observed in the patients showing azoospermia (Table 3).

Table 3. Comparison of the Yqh+, Yqh- and autosomal polymorphic variants in the groups with different sperm counts.

\begin{tabular}{|c|c|c|c|}
\hline \multirow[t]{2}{*}{ Group } & \multicolumn{3}{|c|}{ Cases } \\
\hline & Yqh+ & Yqh- & Autosome Variants \\
\hline Azoospermia & $6(14.2 \%, 6 / 42)$ & $24(57.2 \%$ *\#, 24/42) & $12(28.6 \%, 12 / 42)$ \\
\hline Oligospermia & $8(21.6 \%, 8 / 37)$ & $9\left(24.3 \%^{*}, 9 / 37\right)$ & $20(54.1 \%, 20 / 37)$ \\
\hline Normal sperm count & $3(23.1 \%, 3 / 13)$ & $0(0 \%, 0 / 13)$ & $10(76.9 \%, 10 / 13)$ \\
\hline
\end{tabular}

${ }^{*}$ Compared to the cases showing a normal sperm count, $\mathrm{P}<0.05$. ${ }^{\#}$ Compared to the cases displaying oligospermia, $\mathrm{P}<0.05$.

Tables 4 and 5 summarized the testicular size and plasma hormone levels of the azoospermic and oligospermic patients with chromosomal polymorphic variants in the study and control (II, III, and IV; fertile male individuals with normal karyotype, azoospermic patients with normal karyotype, andoligospermic patients with normal karyotype, respectively) groups. Statistical analyses of the data revealed no differences in the testis volume and testosterone levels between the study and control groups. Interestingly, the follicle stimulating hormone (FSH) and luteinizing hormone (LH) levels did not differ among infertile patients with chromosomal polymorphic variants and those with the normal karyotype; however, the FSH and LH levels were significantly higher in infertile patients than in fertile individuals.

Table 4. Testicular size and plasma hormone levels of chromosomal polymorphic variants of azoospermic patients.

\begin{tabular}{lccc}
\hline Group & Study group & Control group II & Control group III \\
\hline Testis volume Lt $(\mathrm{mL})$ & $14.05 \pm 4.48$ & $15.34 \pm 2.65$ & $14.21 \pm 4.52$ \\
Testis volume Rt $(\mathrm{mL})$ & $14.43 \pm 4.39$ & $15.82 \pm 2.72$ & $14.37 \pm 3.98$ \\
FSH $(\mathrm{mlU} / \mathrm{mL})$ & $17.51 \pm 13.18^{*}$ & $4.11 \pm 1.74$ & $17.11 \pm 17.34$ \\
LH $(\mathrm{mll} / \mathrm{mL})$ & $8.94 \pm 6.03^{*}$ & $3.56 \pm 1.70$ & $8.32 \pm 7.26$ \\
T $(\mathrm{ng} / \mathrm{mL})$ & $4.05 \pm 2.63$ & $4.94 \pm 2.26$ & $4.57 \pm 2.11$ \\
\hline
\end{tabular}

${ }^{*}$ Compared to the control group II, $\mathrm{P}<0.05$. FSH, follicle stimulating hormone; $\mathrm{LH}$, luteinizing hormone; $\mathrm{T}$, testosterone.

Table 5. Testicular size and plasma hormone levels of chromosomal polymorphic variants of oligospermic patients.

\begin{tabular}{lccc}
\hline Group & Study group & Control group II & Control group IV \\
\hline Testis volume Lt $(\mathrm{mL})$ & $14.54 \pm 3.88$ & $15.34 \pm 2.65$ & $14.93 \pm 3.97$ \\
Testis volume Rt $(\mathrm{mL})$ & $14.76 \pm 4.05$ & $15.82 \pm 2.72$ & $15.23 \pm 3.38$ \\
FSH $(\mathrm{ml} / \mathrm{mL})$ & $12.38 \pm 14.23^{*}$ & $4.11 \pm 1.74$ & $8.36 \pm 7.99$ \\
$\mathrm{LH}(\mathrm{ml} / \mathrm{mL})$ & $7.60 \pm 9.86^{*}$ & $3.56 \pm 1.70$ & $6.01 \pm 4.18$ \\
$\mathrm{~T}(\mathrm{ng} / \mathrm{mL})$ & $4.55 \pm 2.03$ & $4.94 \pm 2.26$ & $6.83 \pm 12.73$ \\
\hline
\end{tabular}

${ }^{*}$ Compared with control group II, $\mathrm{P}<0.05$. FSH, follicle stimulating hormone; $\mathrm{LH}$, luteinizing hormone; T, testosterone. 
Samples from 28/50 patients with $\mathrm{Y}$ polymorphic variants were subjected to multiplex PCR amplification; nine of these cases showed detectable Ychromosome microdeletion at a frequency of $32.14 \%$ (9/28). The results of PCR amplification (Table 6) revealed that 8 patients were Yqh-; five and three of these patients displayed azoospermia and oligospermia, respectively. The Yqh+ patient, on the other hand, displayed azoospermia.

Table 6. Y-chromosome microdeletions in eight cases of $Y$ variations.

\begin{tabular}{|c|c|c|c|c|c|c|c|c|c|c|c|}
\hline \multirow[t]{2}{*}{ Case No. } & \multirow[t]{2}{*}{ Karyotype } & \multirow[t]{2}{*}{ Semen analysis } & \multicolumn{2}{|c|}{$\mathrm{AZFa}$} & \multicolumn{3}{|c|}{$\mathrm{AZFb}$} & \multicolumn{4}{|c|}{ AZFc } \\
\hline & & & sY84 & sY86 & sY127 & sY134 & sY143 & sY152 & sY157 & sY254 & sY255 \\
\hline 1 & $46, X Y, Y q h+$ & Azoospermia & + & + & + & + & + & + & + & + & - \\
\hline 2 & 46,XY,Yqh- & Azoospermia & + & + & - & - & - & + & + & + & + \\
\hline 3 & $46, X Y, Y q h-$ & Azoospermia & + & + & + & + & + & - & + & - & - \\
\hline 4 & 46,XY,Yqh- & Oligozoospermia & + & + & + & + & + & - & + & - & - \\
\hline 5 & $46, X Y, Y q h-$ & Azoospermia & + & + & + & + & + & - & - & - & - \\
\hline 6 & $46, X Y, Y q h-$ & Oligozoospermia & + & + & + & + & + & - & - & - & - \\
\hline 7 & 46,XY,Yqh- & Azoospermia & + & + & - & + & + & - & - & - & - \\
\hline 8 & 46,XY,Yqh- & Oligozoospermia & + & + & - & + & + & - & - & - & - \\
\hline 9 & 46,XY,Yqh- & Azoospermia & + & + & - & - & - & - & - & - & - \\
\hline
\end{tabular}

The probands of 35 patients and their parents were subjected to chromosome karyotype analysis (Table 7). Fifteen patients (each) acquired the polymorphic chromosome from their mothers and fathers, while the remaining 5 patients acquired the polymorphic variants by mutation.

Table 7. Pedigree analysis of 35 families with chromosomal polymorphisms.

\begin{tabular}{|c|c|c|c|c|c|}
\hline Case No. & Relationship & Karyotype & Case No. & Relationship & Karyotype \\
\hline \multirow[t]{4}{*}{$1-2$} & Probands & $46, X Y(1 q h+)$ & 12 & Probands & $46, X X(21 p+)$ \\
\hline & Partner & $46, x X$ & & Partner & 46, XY (13pstk+) \\
\hline & Father & 46, XY (1qh+) & & Father & $46, X Y$ \\
\hline & Mother & $46, x x$ & & Mother & 46, XX (21p+) \\
\hline \multirow[t]{4}{*}{3} & Probands & $46, X X(1 \mathrm{gh}+)$ & $13-15$ & Probands & $46, X Y\left(22 s^{+}\right)$ \\
\hline & Partner & $46, X Y$ & & Partner & $46, x x$ \\
\hline & Father & 46, XY (1gh+) & & Father & $46, X Y$ \\
\hline & Mother & $46, x x$ & & Mother & 46, XX (22s+) \\
\hline \multirow[t]{4}{*}{$4-5$} & Probands & $46, X Y(9 q h+)$ & $16-18$ & Probands & $46, X Y \operatorname{inv}(9)(p 11 q 12)$ \\
\hline & Partner & $46, X X$ & & Partner & $46, X X$ \\
\hline & Father & $46, X Y(9 q h+)$ & & Father & $46, X Y, \operatorname{inv}(9)(p 11 q 12)$ \\
\hline & Mother & $46, X X$ & & Mother & $46, X X$ \\
\hline \multirow[t]{4}{*}{6} & Probands & $46, X X(16 q h+)$ & $19-22$ & Probands & $46, X Y, \operatorname{inv}(9)(p 11 q 12)$ \\
\hline & Partner & $46, X Y$ & & Partner & $46, X X$ \\
\hline & Father & $46, X Y$ & & Father & $46, X Y$ \\
\hline & Mother & $46, X X(16 q h+)$ & & Mother & $46, X X, \operatorname{inv}(9)(p 11 q 12)$ \\
\hline \multirow[t]{4}{*}{7} & Probands & 46, XY (16qh+) & 23 & Probands & $46, X Y, \operatorname{inv}(9)(p 11 q 13)$ \\
\hline & Partner & $46, X X$ & & Partner & $46, x X$ \\
\hline & Father & $46, X Y(22 p s t k+)$ & & Father & $46, X Y$ \\
\hline & Mother & 46, $X X(16 \mathrm{gh}+)$ & & Mother & $46, X X, \operatorname{inv}(9)(p 11 q 13)$ \\
\hline \multirow[t]{4}{*}{8} & Probands & $46, X X(15 s+)$ & $24-27$ & Probands & 46, XY $(Y p+)$ \\
\hline & Partner & $46, X Y$ & & Partner & $46, x X$ \\
\hline & Father & $46, X Y$ & & Father & 46, XY (Yp+) \\
\hline & Mother & $46, X X$ & & Mother & $46, X X$ \\
\hline \multirow[t]{4}{*}{9} & Probands & $46, X Y(15 p-)$ & $28-31$ & Probands & 46, XY, Yqh- \\
\hline & Partner & $46, x \times$ & & Partner & $46, x x$ \\
\hline & Father & $46, X Y$ & & Father & 46, XY, Yqh- \\
\hline & Mother & $46, X X(15 p-)$ & & Mother & $46, x X$ \\
\hline \multirow[t]{4}{*}{10} & Probands & $46, X X(21 \mathrm{~s}+)$ & $32-35$ & Probands & 46, XY, Yqh- \\
\hline & Partner & $46, X Y$ & & Partner & $46, X X$ \\
\hline & Father & $46, X Y$ & & Father & $46, X Y$ \\
\hline & Mother & $46, X X\left(21 s^{+}\right)$ & & Mother & $46, X X$ \\
\hline \multirow[t]{4}{*}{11} & Probands & $46, X Y(21$ pstk-) & & & \\
\hline & Partner & $46, X X$ & & & \\
\hline & Father & 46, XY (21pstk-) & & & \\
\hline & Mother & $46, x X$ & & & \\
\hline
\end{tabular}


Among the 23 patients with autosomal polymorphism, 15 and 7 patients acquired the variants from the maternal and paternal sides, respectively; the variant in the remaining patient was obtained by de novo mutation. Eight and four of the 12 patients with polymorphisms in chromosome $\mathrm{Y}$ acquired the variants from their paternal side, and by mutation. Parents of all the patients exhibiting probands with identical chromosomal polymorphisms showed a normal history of reproduction (did not have the same reproductive failure). $Y$ chromosome microdeletions were detected in the fathers of patients displaying probands with microdeletions in the $Y$ chromosome; none of the fathers displayed a $\mathrm{Y}$ chromosome microdeletion.

\section{DISCUSSION}

Several studies conducted over the past few years have investigated the possible association between infertility and selected chromosomal polymorphic variants (Sahin et al., 2008), following a typical development in the published literature. Recent studies have reported higher frequencies of chromosomal polymorphic variants in infertile individuals, compared to the population cytogenetic data (obtained mainly from fertile individual surveys). However, Brothman et al. (2006) reported common cytogenetic variants to be heteromorphic and of no clinical significance. Some studies have suggested that chromosomal polymorphic variants, including quantitative or positional modifications of the constitutive heterochromatin, are associated with male infertility; these variants have also been shown to affect male gamete formation and function (Antonelli et al., 2000). However, polymorphic variants have not yet been proven to be responsible for clinical disorders, such as infertility and behavioral problems.

In this study, the prevalence and proportion of chromosomal polymorphic variants were not significantly different among the fertile and infertile individuals of the study and control I groups (Table 1). However, a significantly greater proportion and higher frequency of chromosome $Y$ variants was observed in the study group, compared to control group I (51.5 vs 26.3; 2.01 vs $0.84 \%$ ). This indicated that the heterochromatin of chromosome $Y$ may play an as yet unknown, significant role in spermatogenesis.

We have also compared the percentage of chromosome $Y$ variants (Yqh+ and Yqh-) and autosomal variants in different sperm counts. The frequency of the polymorphic variant Yqh- was significantly higher in the group of azoospermic patients, while the occurrence of Yqh+ variant did not differ significantly among infertile men and controls. Several studies have suggested that heterochromatin plays an essential role in spindle attachment, chromosomal movement, meiotic pairing, and sister chromatid cohesion (Karpen and Endow, 1998). Microdeletions in the euchromatic region of the $Y$ chromosome are associated with severe male factor infertility (Krausz et al., 2003). The decreased size and occurrence of the Yqh- variant could be associated with the enhancer of gene transcription, due to the enhancing effect on the gene promoter in close proximity. Advances in human genetics, the availability of genetic maps of the $Y$ chromosome, and the mapping of various candidate fertility genes on the long arm of the $Y$ chromosome have revealed that the male-specific region of the human $\mathrm{Y}$ chromosome is a mosaic of polymorphic and euchromatic sequences (Skaletsky et al., 2003). Therefore, polymorphic variants of the $Y$ chromosome could also be associated with severe male factor infertility by inducing epigenetic alterations.

The autosomal polymorphic variants were not (statistically) significantly increased in infertile patients. This result was similar to the results obtained by Kalantari et al. (2000), where the chromosomal and semen analysis of 70 infertile patients revealed that autosomal polymorphism was not significantly associated with spermatogenesis. However, many studies have reported 
conflicting views on the clinical effect of chromosomal variants. Some have reported the presence of variants undervarying clinical conditions, such as reproductive failure (Madon et al., 2005), with higher frequencies of satellite variants having been observed in adults with reproductive failure (Rosenmann et al., 1977). On the other hand, other studies have reported no association between chromosome polymorphisms and recurrent spontaneous abortions (Blumberg et al., 1982), and that variants of the chromosome did not influence the sperm count and fertility of men (Kalantari et al., 2000).

The (statistically) significantly higher FSH and LH levels in the azoospermic and oligospermic patients in our study revealed a possible association between the changes in $\mathrm{LH}$ and $\mathrm{FSH}$ and spermatogenesis and sperm maturation dysfunction in patients with idiopathic azoospermia and oligospermia (Table 4 and Table 5). Al-Daghistani and Abdel-Dayem (2002) reported similar results to ours; that is, infertile patients displayed higher levels of $\mathrm{FSH}$ and LH compared to fertile individuals, while the testosterone level did not differ significantly. However, a comparison of the hormone levels between infertile patients with and without chromosomal polymorphisms revealed no statistically significant differences. This suggested that the chromosomal polymorphic variants were not associated with the regulation of reproductive hormones.

Very few studies have described the association between the variants of, and microdeletions in the $\mathrm{Y}$ chromosome. In this study, the $\mathrm{Y}$ chromosome microdeletion analysis of 28 patients with Yqh \pm revealed the presence of microdeletions in 9 cases (Table 6). The genesin the AZF regions are considered to be critical for spermatogenesis; $Y$ chromosome microdeletions have been associated with the severity of spermatogenic defects (Kato et al., 2001). These microdeletions may explain the infertility of the 9 patients with polymorphisms in the $Y$ chromosome, noted in our study.

In addition, the parents of the 35 individuals displaying probands with chromosomal polymorphisms were subjected to chromosomal analysis and $Y$ chromosome microdeletion analysis, and surveyed their detailed reproductive history. The results revealed that 30 patients acquired the polymorphisms from their parents; we observed a similarity in the karyotypes of the parents with the respective probands in these 30 cases; however, this was not reflected in a similar adverse reproductive history. The fathers of the patients with $\mathrm{Y}$ chromosome microdeletions were subjected to a $Y$ chromosome microdeletion analysis; the results indicated that none of the $Y$ chromosome microdeletions were inherited from the paternal side. The infertility of the probands was suggested to be a result of $Y$ chromosome microdeletions, and not polymorphisms in the $Y$ chromosome.

In conclusion, the results of this study indicated that the association between Yqh- and Y chromosome microdeletions could be responsible for the significantly high frequency of Yqh- in infertile patients; however, the mechanism of this association remains to be elucidated. This results of this study also supported the opinions of previous studies regarding autosomal polymorphisms, that the heterochromatin polymorphisms have no phenotypic or clinical adverse effects, or any apparent association with infertility. We recommend that infertile patients with autosomal polymorphic variants and a normal karyotype can be treated using appropriate reproductive methods; on the other hand, patients with the Yqh \pm karyotype should be diagnosed with a method to detect $Y$ chromosome microdeletions, and treated by choosing female embryos by PGD, in case of the existence of microdeletions.

\section{Conflicts of interest}

The authors declare no conflict of interest. 


\section{ACKNOWLEDGMENTS}

We would like to express our deepest gratitude to the staff of the Genetics and Andrology Laboratories for their excellent work and advice, without which this work would not have been possible. Research supported by the National Population and Family Planning Commission of China (\#2011-GJKJS-07).

\section{REFERENCES}

Al-Daghistani $\mathrm{HI}$ and Abdel-Dayem M (2002). Hyperprolactinemia and hypergonadotropins in infertile males with severe oligospermia and azoospermia. ISPUB 2002: 1540-2606.

Antonelli A, Gandini L, Petrinelli P, Marcucci L, et al. (2000). Chromosomal alterations and male infertility. J. Endocrinol. Invest. 23: $677-683$.

Bhasin MK (2005). Human population cytogenetics: a review. Int. J. Hum. Genet. 5: 83.

Blumberg BD, Shulkin JD, Rotter JI, Mohandas T, et al. (1982). Minor chromosomal variants and major chromosomal anomalies in couples with recurrent abortion. Am. J. Hum. Genet. 34: 948-960.

Brothman AR, Schneider NR, Saikevych I, Cooley LD, et al. (2006). Cytogenetic heteromorphisms: survey results and reporting practices of Giemsa-band regions that we have pondered for years. Arch. Pathol. Lab. Med. 130: 947-949.

Dong Y, Du RC, Jiang YT, Wu J, et al. (2012). Impact of chromosomal translocations on male infertility, semen quality, testicular volume and reproductive hormone levels. J. Int. Med. Res. 40: 2274-2283.

Irvine DS (2002). Andrology: Male reproductive health and dysfunction. Int. J. Androl. 25: 128-128.

Kalantari P, Sepehri H, Behjati F, Ashtiani ZO, et al. (2000). Chromosomal studies in infertile men. Tsitol. Genet. $35:$ 50-54.

Karpen GH and Endow SA (1998). Meiosis: chromosome behavior and spindle dynamics. In: Dynamics of Cell Division (Endow SA and Glover DM, eds.) Oxford University Press, Oxford, 203-236.

Kato H, Komori S, Nakata Y, Sakata K, et al. (2001). Screening for deletions in interval D16-22 of the $Y$ chromosome in azoospermic and oligozoospermic Japanese men. J. Hum. Genet. 46: 110-114.

Krausz C, Forti G and McElreavey K (2003). The Y chromosome and male fertility and infertility. Int. J. Androl. 26: 70-75.

Madon PF, Athalye AS and Parikh FR (2005). Polymorphic variants on chromosomes probably play a significant role in infertility. Reprod. Biomed. Online 11: 726-732.

Mierla D and Stoian V (2012). Chromosomal polymorphisms involved in reproductive failure in the Romanian population. Balkan J. Med. Genet. 15: 23-28.

Poongothai J, Gopenath TS and Manonayaki S (2009). Genetics of human male infertility. Singapore Med. J. 50: $336-347$.

Rosenmann A, Segal S, Palti Z and Cohen MM (1977). Chromosomes in familial primary sterility and in couples with recurrent abortions and stillbirths. Isr. J. Med. Sci. 13: 1131-1133.

Sahin FI, Yilmaz Z, Yuregir OO, Bulakbasi T, et al. (2008). Chromosome heteromorphisms: an impact on infertility. J. Assist. Reprod. Genet. 25: 191-195.

Schaffer LG and Tommerup N (2005). An International System for Human Cytogenetic Nomenclature 2005. Recommendations of the International Standing Committee on Human Cytogenetic Nomenclature. Karger Medical and Scientific Publishers, Seoul.

Skaletsky H, Kuroda-Kawaguchi T, Minx PJ, Cordum HS, et al. (2003). The male-specific region of the human Y chromosome is a mosaic of discrete sequence classes. Nature 423: 825-837.

World Health Organization (2010). WHO laboratory manual for the examination and processing of human semen (5th eds.) World Health Organization, Geneva. 